\title{
Example of q-deformed Field Theory
}

\author{
V.Bardek, M.Dorešić and S.Meljanac円 \\ Department of Theoretical Physics \\ Rudjer Bošković Institute, P.O.B. 1016, \\ 41001 Zagreb,CROATIA
}

\begin{abstract}
The non-relativistic Chern-Simons theory with the single-valued anyonic field is proposed as an example of q-deformed field theory. The corresponding q-deformed algebra interpolating between bosons and fermions, both in position and momentum spaces, is analyzed.A possible generalization to a space with an arbitrary dimension is suggested.
\end{abstract}

PACS numbers: 03.65.-w,03.70.+k,05.30.-d,11.10.Ln.

\footnotetext{
${ }^{1}$ e-mail address: bardek@thphys.irb.hr

e-mail address: doresic@thphys.irb.hr

e-mail address: meljanac@thphys.irb.hr
} 
In the last few years considerable interest has been devoted to non-commutative geometry [1] and quantum groups [2], both in mathematics and physics. The mathematical knowledge is rapidly growing up.On the other hand,the role of these remarkable mathematical structures in physics is still not clear.Moreover,their connection with fundamental physical concepts and laws is missing.

Anyonic physics [3] in the Chern-Simons description can be viewed as a particular realization of these ideas, although in $2+1$ dimensions only. As was shown in [4], the anyonic graded-commutation algebra represents a typical q-deformed structure [5], with q being a unimodular complex parameter,determining the type of intermediate anyonic statistics. For consistency of this approach,it is essential that anyons are multi-valued objects [3].

In this paper we present another type of q-deformed field theory that can be generalized in different directions.We start with the non-relativistic Chern-Simons theory.Using the single-valued transformation we obtain a different type of anyonic field.The corresponding graded-commutation relations for this anyonic field are a generalization of those for quantum-group particles [6]. We point out that our anyonic field is a single-valued object defined on the ordinary $2+1$ space-time. This field is not free and the corresponding commutation relations in momentum space are non-local.Furthemore, we construct $\mathcal{R}$-matrices for anyon operators both in position and momentum space and show that they satisfy the Yang-Baxter equation and the Hecke condition. Finally,we investigate the origin of the transmutation from bosons to anyons ( or fermions to anyons) and suggest a generalization to a space with an arbitrary number of dimensions.

The quantum-mechanical problem of non-relativistic bosons interacting with the U(1) gauge field described by the Chern-Simons Lagrangian has been formulated and solved by Jackiw and Pi [7].They have shown that the corresponding 
Hamiltonian can be reduced to the following form:

$$
H=\frac{1}{2} \int d^{2} r[(\vec{\nabla}-i \vec{A}(\vec{r})) \Psi(\vec{r})]^{\dagger}[(\vec{\nabla}-i \vec{A}(\vec{r})) \Psi(\vec{r})]
$$

The quantum field operator $\Psi(\vec{r})$ and its hermitian conjugate $\Psi^{\dagger}(\vec{r})$ obey the bosonic commutation relations at equal times:

$$
\begin{aligned}
{\left[\Psi(\vec{r}), \Psi\left(\vec{r}^{\prime}\right)\right] } & =0, \\
{\left[\Psi(\vec{r}), \Psi^{\dagger}\left(\vec{r}^{\prime}\right)\right] } & =\delta\left(\vec{r}-\vec{r}^{\prime}\right) .
\end{aligned}
$$

The vector potential $\vec{A}$ can be completely described by the bosonic-number density operator $\rho$ :

$$
\begin{aligned}
\vec{A}(\vec{r}) & =-\lambda \hat{n} \times \int \frac{\vec{r}-\vec{r}^{\prime}}{\left|\vec{r}-\vec{r}^{\prime}\right|^{2}} \rho\left(\vec{r}^{\prime}\right) d^{2} r^{\prime}, \\
\rho(\vec{r}) & =\Psi^{\dagger}(\vec{r}) \Psi(\vec{r})
\end{aligned}
$$

where $\lambda$ is the so-called statistical parameter, and $\hat{n}$ is the unit vector orthogonal to the plane. In other words, the gauge field has no independent dynamics.

Now we introduce a new field $\tilde{\Psi}(\vec{r})$ by performing unitary transformation,i.e. by the phase redefinition of the bosonic field $\Psi(\vec{r})$ :

$$
\tilde{\Psi}(\vec{r})=e^{-i \omega(\vec{r})} \Psi(\vec{r})
$$

where

$$
\omega(\vec{r})=-\lambda \int d^{2} r^{\prime} \theta\left(\vec{r}-\vec{r}^{\prime}\right) \rho\left(\vec{r}^{\prime}\right) .
$$


Here $\theta(\vec{r})$ is the polar angle of the radius vector $\vec{r}$ in respect to the positive $\mathrm{x}$-axis; it is defined as a single-valued function and with $0 \leq \theta(\vec{r})<2 \pi$.In this way we have introduced a cut along the positive $\mathrm{x}$-axis,which induces additional,residual interactions. Then we find that

$$
\vec{\nabla} \omega(\vec{r})=\vec{A}(\vec{r})-\vec{A}_{r e s}(\vec{r})
$$

where the residual vector potential is given by

$$
\vec{A}_{r e s}(\vec{r})=2 \pi \lambda \vec{j} \int_{x}^{\infty} d x^{\prime} \rho\left(x^{\prime}, y\right)
$$

Here $\vec{i}, \vec{j}$ are unit vectors along the positive $\mathrm{x}$ - and $\mathrm{y}$-axis,respectively.In this way we obtain the following Hamiltonian for the new field $\tilde{\Psi}(\vec{r})$ :

$$
H=\frac{1}{2} \int d^{2} r\left[\left(\vec{\nabla}-i \vec{A}_{r e s}(\vec{r})\right) \tilde{\Psi}(\vec{r})\right]^{\dagger}\left[\left(\vec{\nabla}-i \vec{A}_{r e s}(\vec{r})\right) \tilde{\Psi}(\vec{r})\right]
$$

Note that $\vec{B}=\vec{\nabla} \times \vec{A}_{\text {res }}=2 \pi \lambda \hat{n} \rho(\vec{r})$, i.e. that the magnetic field $\vec{B}$ does not change under the transformation (5) .

The commutation relations (2) written in terms of the new field $\tilde{\Psi}(\vec{r})$ are now modified as

$$
\begin{aligned}
& \tilde{\Psi}(\vec{r}) \tilde{\Psi}\left(\vec{r}^{\prime}\right)-e^{-i \lambda \Delta\left(\vec{r}-\vec{r}^{\prime}\right)} \tilde{\Psi}\left(\vec{r}^{\prime}\right) \tilde{\Psi}(\vec{r})=0 \\
& \tilde{\Psi}(\vec{r}) \tilde{\Psi}^{\dagger}\left(\vec{r}^{\prime}\right)-e^{i \lambda \Delta\left(\vec{r}-\vec{r}^{\prime}\right)} \tilde{\Psi}^{\dagger}\left(\vec{r}^{\prime}\right) \tilde{\Psi}(\vec{r})=\delta\left(\vec{r}-\vec{r}^{\prime}\right)
\end{aligned}
$$

where $\Delta$ denotes the difference

$$
\Delta\left(\vec{r}-\vec{r}^{\prime}\right)=\theta\left(\vec{r}-\vec{r}^{\prime}\right)-\theta\left(\vec{r}^{\prime}-\vec{r}\right)
$$




$$
= \begin{cases}-\pi \operatorname{sgn}\left(y-y^{\prime}\right) & \text { if } y-y^{\prime} \neq 0 \\ -\pi \operatorname{sgn}\left(x-x^{\prime}\right) & \text { if } y-y^{\prime}=0\end{cases}
$$

We point out that the antisymmetry of the $\Delta(\vec{r})$ function is a necessary and sufficient condition for the consistency of the above graded-commutation relations.In a multi-valued picture, $\Delta$ is a multi-valued constant,i.e.

$\Delta=\{\pi(1+2 z), z \in Z\}$, satisfying the antisymmetry condition in the sense of the set equality $\Delta=-\Delta$.

In the above approach, the grading factor is not a multi-valued constant but a single-valued function of difference of the appropriate coordinates.

We interpret the $\tilde{\Psi}(\vec{r})$ field as a new type of anyonic field,or the q-deformed field ( quantum-group field). This new field is a generalization of the finite-dimensional quantum spaces with coordinates $\psi_{1}, \bar{\psi}_{1}, \ldots \psi_{n}, \bar{\psi}_{n}$ satisfying the relations [6]

$$
\begin{aligned}
& \psi_{i} \bar{\psi}_{i}=\bar{\psi}_{i} \psi_{i}, \quad 1 \leq i \leq n, \\
& \psi_{i} \psi_{j}=q \psi_{j} \psi_{i}, \quad \bar{\psi}_{i} \bar{\psi}_{j}=q \bar{\psi}_{j} \bar{\psi}_{i} \\
& \psi_{i} \bar{\psi}_{j}=q^{-1} \bar{\psi}_{j} \psi_{i}, \quad \bar{\psi}_{i} \psi_{j}=q \psi_{j} \bar{\psi}_{i}, \quad 1 \leq i<j \leq n, \\
& |q|=1, q^{m}=1 .
\end{aligned}
$$

In this way, finite-dimensional quantum spaces are generalized to infinite-dimensional quantum spaces by the graded- commutation relations (10) . Hence, formally, we have

$$
\psi_{i} \rightarrow \tilde{\Psi}(\vec{r}), \quad \psi_{j} \rightarrow \tilde{\Psi}\left(\vec{r}^{\prime}\right)
$$

and

$$
q \rightarrow e^{-i \lambda \pi}, \quad-\pi \operatorname{sgn}(i-j) \rightarrow \Delta\left(\vec{r}-\vec{r}^{\prime}\right)
$$

The structure of the $2+1$ space-time is not changed.If $\vec{r}=\vec{r}^{\prime}$, the field $\tilde{\Psi}(\vec{r})$ behaves as a bosonic field,whereas for $\vec{r} \neq \vec{r}^{\prime}$,it behaves as a q-deformed field obeying 
fractional statistics for $q \neq \pm 1$. The graded algebra (10) is different from the quon algebra [4, 5].

We note that if we had chosen the fermionic field instead of the bosonic field $\Psi$, as a generic field for anyons in Eq. (5), our q-commutator algebra (10) would have changed the relative sign between bilinears,transforming q-commutators into q-anticommutators. An immediate consequence is the appearance of the hard-core condition for any value of $\lambda$,including even the bosonic case [8]. This can be understood as a counterpart to the absence of any hard-core condition (even for fermions, $\lambda=1$ ) in the bosonic-based approach.Hence, in both approaches, the continuous interpolation between ordinary bosons and fermions is not fully achieved.In fact, the anyonic field, Eqs. (5) and (6), with $\lambda=1$, is the same as the continuum limit of the set of oscillators in Green's ansatz for para-Bose statistics [10].

It is interesting to note that both approaches can be unified into one for $\vec{r} \neq \vec{r}^{\prime}$.Then

$$
e^{i \lambda \Delta}=\cos \lambda \pi+i \frac{\Delta}{\pi} \sin \lambda \pi
$$

The substitution $\lambda=\lambda^{\prime}+1 \bmod 2$ leads to the q-fermionic algebra.This can be viewed as a complexification of the quon algebra of Greenberg [5].

Operating on the vacuum,the anyonic operators $\tilde{\Psi}^{\dagger}(\vec{r})$ create a single-valued quantum state.For example, a two-particle state is given by

$$
\begin{aligned}
\Psi\left(\vec{r}_{1}, \vec{r}_{2}\right) & =\tilde{\Psi}^{\dagger}\left(\vec{r}_{1}\right) \tilde{\Psi}^{\dagger}\left(\vec{r}_{2}\right) \mid 0> \\
& =e^{-i \lambda \theta\left(\vec{r}_{1}-\vec{r}_{2}\right)} \Psi^{\dagger}\left(\vec{r}_{1}\right) \Psi^{\dagger}\left(\vec{r}_{2}\right) \mid 0>
\end{aligned}
$$

We note that this state changes phase by $\pm \lambda \pi$ when two particles are interchanged,depending upon the rotation being clockwise or anticlockwise.The N-particle state can be constructed analogously:

$$
\Psi\left(\vec{r}_{1}, \vec{r}_{2}, \ldots, \vec{r}_{N}\right)=e^{-i \lambda \Sigma_{i<j} \theta\left(\vec{r}_{i}-\vec{r}_{j}\right)} \Psi^{\dagger}\left(\vec{r}_{1}\right) \Psi^{\dagger}\left(\vec{r}_{2}\right) \ldots \Psi^{\dagger}\left(\vec{r}_{N}\right) \mid 0>
$$


The single-valued phase factors in (16) or (17) are a one-dimensional representation of the braid group [9].

Using the q-commutation algebra (10) it is easy to verify that all anyonic states in position space have a non-negative squared norm.

For completeness, we want to formulate and analyze the q-deformed algebra (10) in momentum space.Hence, we should Fourier transform the non-relativistic fields $\Psi(\vec{r})$ and $\tilde{\Psi}(\vec{r})$. The corresponding annihilation operators are

$$
\begin{aligned}
& a(\vec{k})=\frac{1}{2 \pi} \int d^{2} r e^{-i \vec{k} \vec{r}} \Psi(\vec{r}), \\
& \tilde{a}(\vec{k})=\frac{1}{2 \pi} \int d^{2} r e^{-i \vec{k} \vec{r}} \tilde{\Psi}(\vec{r}) .
\end{aligned}
$$

In terms of these operators, the q-deformed algebra (10) translates into

$$
\begin{aligned}
& \tilde{a}(\vec{p}) \tilde{a}(\vec{q})-\frac{1}{4 \pi^{2}} \int d^{2} k \int d^{2} r e^{-i \lambda \Delta(\vec{r})-i \vec{k} \vec{r}} \tilde{a}(\vec{q}+\vec{k}) \tilde{a}(\vec{p}-\vec{k})=0 \\
& \tilde{a}(\vec{p}) \tilde{a}^{\dagger}(\vec{q})-\frac{1}{4 \pi^{2}} \int d^{2} k \int d^{2} r e^{i \lambda \Delta(\vec{r})-i \vec{k} \vec{r}} \tilde{a}^{\dagger}(\vec{q}-\vec{k}) \tilde{a}(\vec{p}-\vec{k})=\delta(\vec{p}-\vec{q}) .
\end{aligned}
$$

Let us use the notation

$$
\frac{1}{4 \pi^{2}} \int d^{2} r e^{i \lambda \Delta(\vec{r})-i \vec{k} \vec{r}}=e^{\frac{i \lambda}{2 \pi} \mathcal{M}(\vec{k})}
$$

where $\mathcal{M}(\vec{k})$ is the Fourier transform of $\Delta(\vec{r})$ :

$$
\begin{aligned}
\mathcal{M}(\vec{k}) & =\frac{1}{2 \pi} \int d^{2} r e^{-i \vec{k} \vec{r}} \Delta(\vec{r}) \\
& =2 \pi i \delta\left(k_{x}\right) \mathcal{P} \frac{1}{k_{y}}
\end{aligned}
$$

Then we find that

$$
e^{\frac{i \lambda}{2 \pi} \mathcal{M}(\vec{k})}=\cos (\pi \lambda) \delta(\vec{k})-\frac{1}{\pi} \sin (\pi \lambda) \delta\left(k_{x}\right) \mathcal{P} \frac{1}{k_{y}}
$$


Hence, the q-commutation relations in momentum space transform into

$$
\begin{aligned}
\tilde{a}(\vec{p}) \tilde{a}(\vec{q}) & -\cos (\pi \lambda) \tilde{a}(\vec{q}) \tilde{a}(\vec{p}) \\
& -\frac{1}{\pi} \sin (\pi \lambda) \mathcal{P} \int_{-\infty}^{\infty} \frac{d k}{k} \tilde{a}(\vec{q}+\vec{j} k) \tilde{a}(\vec{p}-\vec{j} k)=0 \\
\tilde{a}(\vec{p}) \tilde{a}^{\dagger}(\vec{q}) & -\cos (\pi \lambda) \tilde{a}^{\dagger}(\vec{q}) \tilde{a}(\vec{p}) \\
+ & \frac{1}{\pi} \sin (\pi \lambda) \mathcal{P} \int_{-\infty}^{\infty} \frac{d k}{k} \tilde{a}^{\dagger}(\vec{q}-\vec{j} k) \tilde{a}(\vec{p}-\vec{j} k)=\delta(\vec{p}-\vec{q}) .
\end{aligned}
$$

If $\sin (\pi \lambda) \neq 0$, there is a continuum of contributions along the $k_{y}$ momentum. This non-locality in momentum space is a consequence of the $\vec{r}$-dependence in the grading factors in (10), i.e. $\Delta(\vec{r}) \neq$ const .

Closer inspection of Eq. (23) at the point of coincidence, i.e. $\vec{p}=\vec{q}$, shows that the fermionic field $(\lambda=1 \bmod 2)$ indeed satisfies the expected hard-core condition. For intermediate $\lambda, \sin (\pi \lambda) \neq 0$, a new unexpected "sum rule" emerges for the operator bilinears at the same point in momentum space :

$$
\tilde{a}^{2}(\vec{p})(1-\cos (\pi \lambda))-\frac{\sin (\pi \lambda)}{\pi} \mathcal{P} \int_{-\infty}^{\infty} \frac{d k}{k} \tilde{a}(\vec{p}+\vec{j} k) \tilde{a}(\vec{p}-\vec{j} k)=0 .
$$

No hard-core condition appears directly, even if we start with the q-anticommutator algebra in position space.

The connection between the momentum operators $a(\vec{k})$ and $\tilde{a}(\vec{k})$ is

$$
\begin{aligned}
\tilde{a}(\vec{k}) & =\frac{1}{2 \pi} \int d^{2} r e^{-i \vec{k} \vec{r}-i \omega(\vec{r})} \Psi(\vec{r}) \\
& =\int d^{2} k^{\prime} e^{-\frac{i}{2 \pi} \tilde{\omega}\left(\vec{k}-\vec{k}^{\prime}\right)} a\left(\vec{k}^{\prime}\right),
\end{aligned}
$$

where

$$
\tilde{\omega}(\vec{k})=-2 \pi \lambda \tilde{\theta}(\vec{k}) \tilde{\rho}(\vec{k})
$$

and

$$
\tilde{\theta}(\vec{k})=\frac{1}{2 \pi} \int d^{2} r e^{-i \vec{k} \vec{r}} \theta(\vec{r})
$$




$$
\begin{aligned}
& =2 \pi \delta(\vec{k})+\frac{1}{k^{2}} \tan \alpha+\frac{1}{2} \mathcal{M}(\vec{k}), \\
\tilde{\rho}(\vec{k}) & =\frac{1}{2 \pi} \int d^{2} r e^{-i \vec{k} \vec{r}} \rho(\vec{r}) \\
& =\frac{1}{2 \pi} \int d^{2} k^{\prime} \tilde{a}^{\dagger}\left(\vec{k}^{\prime}\right) \tilde{a}\left(\vec{k}+\vec{k}^{\prime}\right) .
\end{aligned}
$$

Here $\alpha$ is the polar angle between the $\vec{k}$ and the positive $k_{x}$ axis, and $\mathcal{M}(\vec{k})$ is given by Eq. (21). Hence

$$
\begin{aligned}
\tilde{a}(\vec{k})= & a(\vec{k})+i \lambda \int d^{2} k^{\prime} \tilde{\theta}\left(\vec{k}-\vec{k}^{\prime}\right) \tilde{\rho}\left(\vec{k}-\vec{k}^{\prime}\right) a\left(\vec{k}^{\prime}\right) \\
& +\frac{(i \lambda)^{2}}{2 !} \int d^{2} k^{\prime} d^{2} k^{\prime \prime}[\tilde{\theta} \tilde{\rho}]\left(\vec{k}-\vec{k}^{\prime \prime}\right)[\tilde{\theta} \tilde{\rho}]\left(\vec{k}^{\prime \prime}-\vec{k}^{\prime}\right) a\left(\vec{k}^{\prime}\right)+\ldots,
\end{aligned}
$$

where we have formally expanded the exponential kernel in Eq. (25) in powers of the statistical parameter $\lambda$.

Now we can use the conventional Fock-space method to construct multiparticle states in momentum space by acting of the $\tilde{a}^{\dagger}(\vec{p})$ operators on the vacuum. For a two-particle state,for example,this yields

$$
\tilde{a}^{\dagger}\left(\vec{p}_{1}\right) \tilde{a}^{\dagger}\left(\vec{p}_{2}\right)\left|0>=\frac{1}{2 \pi} \int d^{2} k \int d^{2} r e^{-i \lambda \theta(\vec{r})-i \vec{k} \vec{r}} a^{\dagger}\left(\vec{p}_{1}+\vec{k}\right) a^{\dagger}\left(\vec{p}_{2}-\vec{k}\right)\right| 0>.
$$

It is not obvious that this complicated structure, in contrast to the corresponding structure given by Eq. (17) in position space,possesses the braiding property. However,braiding is still present. To show this,let us mention that q-commutator algebras both in position space Eqs. (10), (11) and in momentum space Eqs. (23) can be written in the $\mathcal{R}$-matrix approach [11]:

$$
\begin{aligned}
\tilde{\Psi}\left(\vec{r}_{1}\right) \tilde{\Psi}\left(\vec{r}_{2}\right)-\int d^{2} x \int d^{2} y \mathcal{R}\left(\vec{r}_{1}, \vec{r}_{2}, \vec{x}, \vec{y}\right) \tilde{\Psi}(\vec{y}) \tilde{\Psi}(\vec{x}) & =0 \\
\tilde{\Psi}\left(\vec{r}_{1}\right) \tilde{\Psi}^{\dagger}\left(\vec{r}_{2}\right)-\int d^{2} x \int d^{2} y \mathcal{R}\left(\vec{y}, \vec{r}_{1}, \vec{r}_{2}, \vec{x}\right) \tilde{\Psi}^{\dagger}(\vec{y}) \tilde{\Psi}(\vec{x}) & =\delta\left(\vec{r}_{1}-\vec{r}_{2}\right) .
\end{aligned}
$$

The $\mathcal{R}$-matrix in position space is given by

$$
\mathcal{R}\left(\vec{r}_{1}, \vec{r}_{2}, \vec{r}_{3}, \vec{r}_{4}\right)=e^{-i \lambda \Delta\left(\vec{r}_{1}-\vec{r}_{2}\right)} \delta\left(\vec{r}_{1}-\vec{r}_{3}\right) \delta\left(\vec{r}_{2}-\vec{r}_{4}\right)
$$


and the $\mathcal{R}$-matrix in momentum space is given by

$$
\begin{aligned}
& \mathcal{R}\left(\vec{k}_{1}, \vec{k}_{2}, \vec{k}_{3}, \vec{k}_{4}\right)=\delta\left(\vec{k}_{3}+\vec{k}_{4}-\vec{k}_{1}-\vec{k}_{2}\right) \\
& \quad \times\left[\cos (\pi \lambda) \delta\left(\vec{k}_{1}-\vec{k}_{3}\right)+\frac{1}{\pi} \sin (\pi \lambda) \delta\left(k_{3 x}-k_{1 x}\right) \mathcal{P} \frac{1}{k_{3 y}-k_{1 y}}\right] .
\end{aligned}
$$

In order that the above $\mathcal{R}$-matrix algebra be associative, the following conditions have to be satisfied:

(i) Yang-Baxter equation:

$$
\begin{aligned}
& \iiint d^{2} x d^{2} y d^{2} z \mathcal{R}\left(\vec{r}_{1}, \vec{r}_{2}, \vec{x}, \vec{y}\right) \mathcal{R}\left(\vec{y}, \vec{z}, \vec{r}_{3}, \vec{r}_{4}\right) \mathcal{R}\left(\vec{x}, \vec{r}_{5}, \vec{r}_{6}, \vec{z}\right) \\
& =\iiint d^{2} x d^{2} y d^{2} z \mathcal{R}\left(\vec{r}_{2}, \vec{r}_{5}, \vec{x}, \vec{y}\right) \mathcal{R}\left(\vec{z}, \vec{x}, \vec{r}_{6}, \vec{r}_{3}\right) \mathcal{R}\left(\vec{r}_{1}, \vec{y}, \vec{z}, \vec{r}_{4}\right)
\end{aligned}
$$

(ii) hermiticity:

$$
\mathcal{R}\left(\vec{r}_{1}, \vec{r}_{2}, \vec{r}_{3}, \vec{r}_{4}\right)=\mathcal{R}^{*}\left(\vec{r}_{4}, \vec{r}_{3}, \vec{r}_{2}, \vec{r}_{1}\right)
$$

(iii) Hecke condition

$$
\iint d^{2} x d^{2} y \check{\mathcal{R}}\left(\vec{r}_{1}, \vec{r}_{2}, \vec{x}, \vec{y}\right) \check{\mathcal{R}}\left(\vec{x}, \vec{y}, \vec{r}_{3}, \vec{r}_{4}\right)=\delta\left(\vec{r}_{1}-\vec{r}_{3}\right) \delta\left(\vec{r}_{2}-\vec{r}_{4}\right)
$$

or symbolically:

$$
(\check{\mathcal{R}}-1)(\check{\mathcal{R}}+1)=0
$$

where

$$
\begin{aligned}
\check{\mathcal{R}} & =P \mathcal{R} \quad \text { and } \\
P\left(\vec{r}_{1}, \vec{r}_{2}, \vec{r}_{3}, \vec{r}_{4}\right) & =\delta\left(\vec{r}_{1}-\vec{r}_{4}\right) \delta\left(\vec{r}_{2}-\vec{r}_{3}\right)
\end{aligned}
$$

It is straightforward to show that the $\mathcal{R}$-matrices (32) and (33) satisfy all the above conditions both in position and momentum spaces. Hence this proves the braiding properties both in position and momentum spaces. 
In order to analyze the origin of the q-deformed algebra (10), we introduce a new field $\Phi(\vec{r})$ defined as

$$
\Phi(\vec{r})=e^{-i \omega^{\prime}(\vec{r})} \Psi(\vec{r})
$$

with

$$
\omega^{\prime}(\vec{r})=-\lambda \int d^{2} r^{\prime} \arctan \frac{y-y^{\prime}}{x-x^{\prime}} \rho\left(\vec{r}^{\prime}\right)
$$

Then,

$$
\vec{\nabla} \omega^{\prime}(\vec{r})=\vec{A}(\vec{r})-\vec{A}_{r e s}^{\prime}(\vec{r})
$$

where

$$
\vec{A}_{r e s}^{\prime}(\vec{r})=\pi \lambda \vec{i} \int_{-\infty}^{\infty} d y^{\prime} \operatorname{sgn}\left(y-y^{\prime}\right) \rho\left(x, y^{\prime}\right)
$$

The $\vec{A}_{\text {res }}^{\prime}$ potential originates from the cut along the y-axis.

The field $\Phi(\vec{r})$ remains bosonic,satisfying the same commutation relations as $\Psi(\vec{r})$ in Eq. (2) .This follows from the fact that $\arctan \frac{y}{x}$ is a symmetric function under the inversion $\vec{r} \rightarrow-\vec{r}$.Hence, $\Delta(\vec{r})=0$. The Hamiltonian of the new bosonic field $\Phi(\vec{r})$ is not free because of ${\overrightarrow{A_{r e s}^{\prime}}}^{\prime}$. Note that $\vec{B}=\vec{\nabla} \times{\overrightarrow{A_{r e s}^{\prime}}}^{\prime}=2 \pi \lambda \hat{n} \rho(\vec{r})$, i.e. the magnetic field does not change under the transformation (37). Hence all the physical consequences are the same as for the initial non-relativistic Chern-Simons theory.

Finally, we connect the q-deformed anyonic field $\tilde{\Psi}(\vec{r})$ with the bosonic field $\Phi(\vec{r})$ :

$$
\tilde{\Psi}(\vec{r})=e^{-i\left[\omega(\vec{r})-\omega^{\prime}(\vec{r})\right]} \Phi(\vec{r})
$$

where $\omega(\vec{r})$ and $\omega^{\prime}(\vec{r})$ are given by (6) and (38), respectively.

The effective kernel in the expression $\omega(\vec{r})-\omega^{\prime}(\vec{r})$ is

$$
\begin{aligned}
\theta_{e f f}(\vec{r}) & =\theta(\vec{r})-\arctan \left(\frac{y}{x}\right) \\
& = \begin{cases}\pi-\frac{\pi}{2} \operatorname{sgn}(x y)-\frac{\pi}{2} \operatorname{sgn}(y) & \text { if } y \neq 0 \\
\frac{\pi}{2}(1-\operatorname{sgn}(x)) & \text { if } y=0 .\end{cases}
\end{aligned}
$$


It is important to note that only the antisymmetric part of $\theta_{\text {eff }}$ deforms the commutation relations,yielding $\Delta(\vec{r})$ given by Eq. (11). The effective kernel $\theta_{\text {eff }}$ changes the cut along the positive $\mathrm{x}$-axis into the cut along the $\mathrm{y}$-axis, and vice versa.Hence $\theta_{\text {eff }}$ relates $\vec{A}_{\text {res }}$ to $\vec{A}_{\text {res }}^{\prime}$.

The origin of the transmutation from bosons to anyons ( or fermions to anyons ) is in the antisymmetric part of the angle function. This suggests a generalization of the above construction for a two-dimensional space to a space $\mathcal{R}^{n}$ with an arbitrary number of dimensions $n$.

Let $x=\left(x_{1}, \ldots x_{n}\right) \in \mathcal{R}^{n}$, then we define

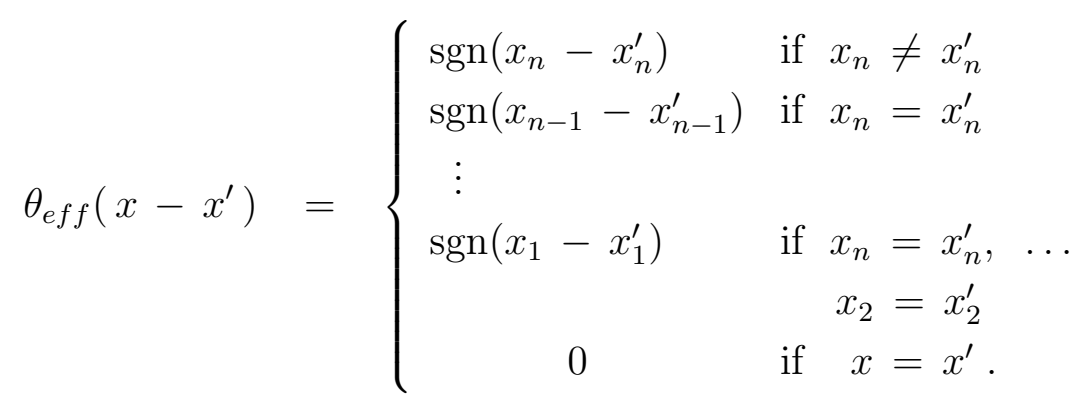

The interpolation between bosons and fermions is achieved by $\tilde{\Psi}(\vec{r})$ given by Eqs. (5), (6) and (43) .

The N-particle anyonic wave function for $\vec{r}_{1} \neq \vec{r}_{2} \neq \ldots \neq \vec{r}_{N}$ has a simple form expressed in terms of the bosonic field $\Psi(\vec{r})$,

$$
\begin{aligned}
& \tilde{\Psi}^{\dagger}\left(\vec{r}_{i_{1}}\right) \tilde{\Psi}^{\dagger}\left(\vec{r}_{i_{2}}\right) \ldots \tilde{\Psi}^{\dagger}\left(\vec{r}_{i_{N}}\right) \mid 0>= \\
& =e^{-i \lambda \pi \Sigma_{\alpha<\beta} \theta_{e f f}\left(\vec{r}_{i_{\alpha}}-\vec{r}_{i_{\beta}}\right)} \Psi^{\dagger}\left(\vec{r}_{1}\right) \Psi^{\dagger}\left(\vec{r}_{2}\right) \ldots \Psi^{\dagger}\left(\vec{r}_{N}\right) \mid 0> \\
& =e^{i \lambda 2 \pi\left[\frac{N(N-1)}{4}-P\left(i_{1}, \ldots i_{N}\right)\right]} \Psi^{\dagger}\left(\vec{r}_{1}\right) \Psi^{\dagger}\left(\vec{r}_{2}\right) \ldots \Psi^{\dagger}\left(\vec{r}_{N}\right) \mid 0>
\end{aligned}
$$

Here $i_{1}, \ldots i_{N}$ is the permutation of indices $1,2, \ldots N$, and $P\left(i_{1}, \ldots i_{N}\right)$ denotes the number of inversions in permutation $i_{1}, \ldots i_{N}$ in respect to the normal order $1,2, \ldots N$, defined by $\theta_{\text {eff }}\left(\vec{r}_{\alpha}-\vec{r}_{\beta}\right)=+1$ iff $\alpha>\beta$. The braiding properties 
hold both in position and momentum space,since the corresponding $\mathcal{R}$-matrices satisfy the Yang-Baxter equation and the Hecke condition. The properties of the free Hamiltonian with such a q-deformed field are under investigation.

\section{Acknowledgment}

This work was supported by the Scientific Fund of the Republic of Croatia. 


\section{References}

[1] A. Connes,Publ.Math. I. H. E. S.,no 62.Non-Commutative Differential Geometry,(1986).

[2] L. D. Faddeev, N. Y. Reshetikhin and L. A. Takhtajan,Quantization of Lie Groups and Lie Algebras, preprint LOMI (1987).

[3] G. Semenoff and P. Sodano, Nucl.Phys. B 328,753 (1989).

[4] V. Bardek,M. Dorešić and S. Meljanac,submitted to Phys.Rev.D .

[5] O. W. Greenberg,Phys.Rev.Lett.64, 705 (1990); Phys.Rev.D43, 4111 (1991).

[6] N. V. Borisov, K. N. Ilinski and V. M. Uzdin,Phys.Lett.A 169,427 (1992).

[7] R. Jackiw and S. Y. Pi,Phys.Rev.D42,3500 (1990).

[8] A. Lerda and S. Sciuto,Torino preprint DFTT 73/92.

[9] Y.-S.Wu,Phys.Rev.Lett.52, 2103 (1984).

[10] Y.Ohnuki and S.Kamefuchi,Quantum Field Theory and Parastatistics (University of Tokyo Press, Tokyo;Springer,Berlin,1982).

[11] D.B.Fairlie and C.K.Zachos,Phys.Lett.256B, 43 (1991). 